\title{
Comments on "Measurement of the production of charged pions by protons on a tantalum target"
}

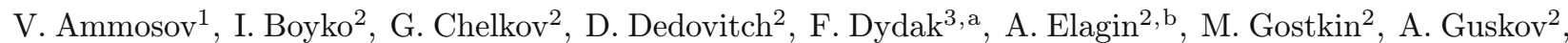 \\ V. Koreshev' ${ }^{1}$, Z. Kroumchtein ${ }^{2}$, Yu. Nefedov², K. Nikolaev' , J. Wotschack ${ }^{3}$, A. Zhemchugov ${ }^{2}$ \\ ${ }^{1}$ Institute of High Energy Physics (IHEP), 142284 Protvino, Russia \\ 2 Joint Institute for Nuclear Research (JINR), 141980 Dubna, Russia \\ 3 CERN, 1211 Geneva 23, Switzerland
}

Received: 3 August 2007 / Revised version: 19 October 2007 /

Published online: 26 January 2008 - (c) Springer-Verlag / Società Italiana di Fisica 2008

\begin{abstract}
We argue that the cross-sections reported in the article "Measurement of the production of charged pions by protons on a tantalum target" by M.G. Catanesi et al., published in this journal, result from invalid analysis concepts and should not be trusted.
\end{abstract}

PACS. 13.85. Ni; 25.40.Ep

\section{Introduction}

The larger part of the HARP Collaboration (hereafter referred to as 'authors') published in this journal an article (hereafter referred to as 'article') entitled 'Measurement of the production of charged pions by protons on a tantalum target' [1]. This article has not been signed by us, also members of the HARP Collaboration, because we feel unable to take responsibility for the reported results.

The detectors used for the analysis are the time projection chamber (TPC) and the resistive plate chambers (RPCs) that surround it. These 'Comments' refer to statements and claims about these detectors in the article, and before in $[2-7]$.

When measuring cross-sections of $\pi^{+}$and $\pi^{-}$production by hadrons on nuclei, the prime concerns are momentum scale, momentum resolution, and particle identification. After an explanation of the core issue at stake, we shall argue that in the authors' analysis the momentum scale is biased, the momentum resolution is worse than claimed, and particle identification is poor.

\section{The core issue}

The performance of the HARP TPC was plagued, inter alia, by dynamic track distortions that were primarily, but not solely, caused by the build-up of an $\mathrm{Ar}^{+}$ion cloud

\footnotetext{
${ }^{\text {a }}$ e-mail: friedrich.dydak@cern.ch

b Present address: Texas A\&M University, College Station, USA
}

during the $400 \mathrm{~ms}$ long spill of the CERN Proton Synchrotron. This ion cloud emanates from the TPC's sense wires and drifts across its active volume toward the highvoltage membrane. The cause of this hardware problem, the physics of the track distortions, their quantitative assessment, and their corrections, are described in [8-11].

To a good approximation, these dynamic track distortions increase linearly with time in the spill ${ }^{1}$. Their size in the $r \cdot \phi$ coordinate typically reaches $15 \mathrm{~mm}$, at small radius, at the end of the spill. That exceeds the TPC's design $r \cdot \phi$ resolution of $500 \mu \mathrm{m}$ by a factor of 30 and hence requires a $\sim 2 \%$ precision of the correction.

The authors chose to use only the first 100 events out of typically 300 events in the whole accelerator spill. From the 'physics benchmark' of proton-proton elastic scattering they claim that dynamic distortions do not affect the quality of the first 100 events. In other words, they claim that a distortion that has grown by the 100th event to $5 \mathrm{~mm}$ at small radius, does not appreciably affect their results and hence needs no corrective action.

In the HARP TPC, with a positive magnetic field polarity, dynamic distortions shift cluster positions such that positive tracks are biased toward higher $p_{\mathrm{T}}$ (conversely, negative tracks are biased to smaller $p_{\mathrm{T}}$ ). The authors chose - in principle correctly - to fit TPC tracks with the constraint of the beam point because the increased lever arm almost doubles the $p_{\text {T }}$ precision. While the cluster pos-

\footnotetext{
1 The increase is not strictly linear because the distortion from the ion cloud saturates when a track gets immersed in the cloud; a second, smaller, distortion is also present that starts nearly instantaneously and causes a constant offset throughout the spill.
} 
itions get shifted, the beam point remains unaffected. Assigning a sufficiently small position error of the beam point renders its weight (the inverse error squared) in the track fit so large that positive tracks get biased toward lower $p_{\mathrm{T}}$, i.e., the trend of the bias is reversed with respect to the fit without beam point. This - artificially enforced - decrease of the $p_{\mathrm{T}}$ of positive tracks with the time in the spill is demonstrated in the article's Fig. 15 (right panel).

Evidently, there must be a weight of the beam point which keeps $p_{\mathrm{T}}$ on the average more or less constant during the spill. This consideration makes clear that the weight assigned to the beam point is of decisive importance.

Four observations on this analysis concept are in order.

First, despite its decisive importance, nowhere in the authors' papers is the position error of the beam point as used in track fits stated.

Second, the position error of the beam point cannot be chosen as deemed suitable. It is objectively determined for each individual track. The position error of the beam point to be used in the fit is the convolution of the errors of two extrapolations to the interaction vertex: of the beam particle's trajectory and of the secondary track's trajectory; primarily because of the momentum-dependence of multiple scattering, the overall error varies considerably for different beam momenta and from track to track. This error is logically different from an 'error' that artificially counters effects from dynamic distortions.

Third, a helix fit of points that deviate systematically from the correct helical trajectory, and/or wrong position errors of the points, cannot give an unbiased $p_{\mathrm{T}}$ estimate.

Fourth, the authors' justification of their analysis concept that it forces later events in the spill to yield the same momentum as the very first events, does not hold. Because of the wrong weight assigned to the beam point, the fit of $p_{\mathrm{T}}$ is already biased at the start of the spill. Because of the distortion already present for the very first events in the spill, their $p_{\mathrm{T}}$ is not correctly measured. The relevant statistical error would be the one from the very first events only.

There is no 'plateau' as conjectured in [2, Sect. 5.1.7.6.], i.e., a retardation of dynamic distortions at the start of the spill. A retardation of dynamic distortions beyond $\sim 1 \mathrm{~ms}$ is in conflict with the strong electric field gradient between the TPC's sense- and grid-wire electrodes. Incidentally, a practically immediate linear increase of dynamic distortions with time in the spill is confirmed by the article's Fig. 11 (left panel).

One cannot talk of 'the' dynamic distortion. Rather, dynamic distortions depend on beam energy, beam polarity, beam intensity, beam scraping, target type, photon conversion in materials, spiralling low-momentum electrons, and sense wire amplification. Therefore, they appear as rather erratic. However, they can be well understood from first principles. Their correction depends on a variety of parameters of which the density and the radial distribution of the ion cloud are most important. Quantitative conclusions from one data set cannot be applied to another.

In particular, it is incorrect to apply quantitative conclusions on dynamic distortions from data taken with a hy- drogen target to data taken with a Ta target, as is done in the article.

The article's parameter $d_{0}^{\prime}$ which is claimed to characterize the strength of track distortions ${ }^{2}$, is inadequate. A non-zero $d_{0}^{\prime}$ merely signifies the existence of track distortions. The highly non-trivial dependence of dynamic distortions on radius and on the $z$ coordinate across the TPC volume cannot be condensed into one single parameter.

The parameter $d_{0}^{\prime}$ is quantitatively meaningful only when it is consistent with zero ${ }^{3}$ as a function both of $p_{\mathrm{T}}$ and polar angle $\theta$. A $d_{0}^{\prime}$ averaged over $p_{\mathrm{T}}$ and $\theta$ has no meaning. Constancy of $d_{0}^{\prime}$ throughout the spill has no meaning either.

In the following we focus on the most serious problem in the article's Ta data analysis. We explain why and how the authors' treatment of TPC track distortions leads to crosssections that should not be trusted.

There are many more mistakes in the authors' data analysis. For a comprehensive discussion we refer to [12] and [13], and especially to [14].

\section{On the momentum scale}

Qualitatively, what happens in the presence of dynamic track distortions is that the $p_{\mathrm{T}}$ scale acquires a bias that increases about linearly with the time in the spill. This bias can be viewed as a pedestal to the track sagitta. Therefore, the bias is most manifest at large $p_{\mathrm{T}}$ and its effect on $p_{\mathrm{T}}$ changes sign with the particle charge.

Quantitively, the bias depends on the weight of the beam point used in the track fit. Since this has never been specified by the authors we must resort to other means to estimate their $p_{\mathrm{T}}$ bias.

In this section, we shall discuss evidence from $\mathrm{d} E / \mathrm{d} x$ distributions. At the end of Sect. 5, we shall discuss supporting evidence from RPC time of flight.

We recall that the authors claim in their Sect. 5.2 a bias of less than $3 \%$ on the absolute momentum scale of positive particles. We shall argue that their momentum scale of positive particles is wrong by $20 \%$ at $p=800 \mathrm{MeV} / \mathrm{c}$.

The claim on the precision of the momentum scale of positive particles is based on the 'physics benchmark' of elastic proton-proton scattering, shown in Fig. 13 (right panel). The conclusion 'In the absence of a clear trend one concludes that the bias is less than $3 \%$ ' is not valid for four reasons.

First, the conclusion is based on hydrogen data and is therefore not applicable to Ta data. Second, their plot is limited to the first 50 rather than the first 100 events in the spill and is therefore biased toward 'better' data. Third, their plot is based on a fit without the beam point which addresses an entirely different problem with respect

\footnotetext{
$2 d_{0}^{\prime}$ is the signed closest distance of approach between the extrapolated trajectory of the beam particle and the extrapolated fit without beam point of a secondary track.

3 Neglecting second-order effects that arise from finite experimental resolution.
} 
to dynamic distortions than the reported Ta data that employ a fit with the beam point. Fourth, their 'physics benchmark' is limited to positive tracks with a polar angle around $70^{\circ}$ out of $20-135^{\circ}$, and which sample a mere $20 \mathrm{~cm}$ out of the $150 \mathrm{~cm}$ long active TPC volume; even if this benchmark were passed successfully, one could not claim the understanding of the whole active TPC volume for Ta data, let alone any understanding of negative tracks.

The authors contradict themselves in Fig. 17 (upper left panel) where they show the measurement of the specific ionization $\mathrm{d} E / \mathrm{d} x$ of protons as a function of momentum ${ }^{4}$. One only needs to overlay the theoretical prediction, well visible in the three other panels, on the proton entries. One reads off that an $800 \mathrm{MeV} / c$ proton is measured with a momentum of $650 \mathrm{MeV} / c$.

The authors contend that the comparison of the $\mathrm{d} E / \mathrm{d} x$ data is made with a simple $1 / \beta^{2}$ prediction rather than with the Bethe-Bloch formula. However, even with the Bethe-Bloch formula it is impossible to describe simultaneously their $\mathrm{d} E / \mathrm{d} x$ of protons and pions. We also note the distinct difference from [2, Fig. 13], where protons observed in cosmic rays and measured without dynamic distortions follow much better the theoretical prediction.

The momentum bias in the authors' Ta analysis, expressed as a shift of $1 / p_{\mathrm{T}}$, is $\Delta\left(1 / p_{\mathrm{T}}\right) \sim 0.3(\mathrm{GeV} / c)^{-1}$. It is of the same size as the claimed resolution in $1 / p_{\mathrm{T}}$.

We recall that the authors claim in Sect. 5.3 that the momentum scale of negative particles is asymmetric at the $1 \%$ level with respect to that of positive particles. We shall argue that the asymmetry is $40 \%$ at $p=800 \mathrm{MeV} / c$.

The 1\% claim stems from Fig. 15 (left panel) which shows the average reconstructed momentum of $\pi^{+}$and $\pi^{-}$ in a variety of targets as a function of the event number in the spill. First, it remains unclear how the dynamic distortions in all these targets are related to dynamic distortions specifically in the Ta data. Second, the plot suggests constancy until event number 200 and no difference between $\pi^{+}$and $\pi^{-}$; the pion momentum is $100 \mathrm{MeV} / c$; a momentum bias of $20 \%$ at $800 \mathrm{MeV} / c$ translates into a bias of $2.5 \%$ at $100 \mathrm{MeV} / c$; the resulting decrease of momentum for one charge sign, and increase of momentum for the other charge sign, would hardly be visible in Fig. 15.

Yet the authors claim that their analysis is correct up to a momentum of $800 \mathrm{MeV} / c$. It remains unclear how one can deduce from a plot at $100 \mathrm{MeV} / c$ that can hardly show the expected defect, the absence of a defect at $800 \mathrm{MeV} / c$, with a precision of $1 \%$.

From the $20 \%$ scale error for positive particles at $p=$ $800 \mathrm{MeV} / c$ which stems from the neglect of track distortions, we conclude that a scale error of $20 \%$ in the opposite direction exists for negative particles. The momentum scales of positive and negative particles differ by $40 \%$ at $p=800 \mathrm{MeV} / c$.

The article's limitation of differential cross-sections to the range $100-800 \mathrm{MeV} / c$ lacks any physics justifi-

\footnotetext{
4 We note that the authors assume implicitly in their argumentation a $\mathrm{d} E / \mathrm{d} x$ that is independent of the polar angle $\theta$; while this is theoretically expected, we conjecture that it is not the case for their measured $\mathrm{d} E / \mathrm{d} x$.
}

cation. First, we note that the real cut applied in the article's results is $p_{\mathrm{T}}<500 \mathrm{MeV} / c$, not $p<800 \mathrm{MeV} / c$ as misleadingly stated. Second, the implicit motivation of this limitation by ongoing design studies for the neutrino factory does not justify the publication of questionable results when, with some further investment of effort, perfectly good data including large $p_{\mathrm{T}}$ could be published.

\section{On the momentum resolution}

The article's Fig. 9 claims a resolution $\sigma\left(1 / p_{\mathrm{T}}\right)$ of $\sim 0.3(\mathrm{GeV} / c)^{-1}$ both for cosmic-ray muons and for physics tracks. We shall argue that the actual resolution is much worse.

First we address the momentum resolution of cosmicray muons. These tracks are recorded in special runs and are not affected by dynamic distortions. Hence the resolution for cosmic-ray muons has a priori no meaning for physics data. It is even more meaningless when - as is the case - the precision of the 'middle point' of through-going cosmic-ray muons is not specified, which is the equivalent of the 'beam point' for physics tracks.

Now we address the momentum resolution of physics tracks. Table 1 gives the expected resolution $\sigma\left(1 / p_{\mathrm{T}}\right)$ for an $r \cdot \phi$ error of a cluster of $1.5 \mathrm{~mm}^{5}$, for fits without and with the beam point, and for different assumptions on the position error of the beam point. We note that with an $r \cdot \phi$ error of $1.5 \mathrm{~mm}$, a resolution $\sigma\left(1 / p_{\mathrm{T}}\right)$ of $\sim 0.3(\mathrm{GeV} / c)^{-1}$ is only possible with a position error of the beam point in the range $1-2 \mathrm{~mm}$. With a $5 \mathrm{~mm}$ error of the beam point, the resolution hardly improves. Hence the error that is assigned to the beam point is decisive not only for the momentum scale but also for the $1 / p_{\mathrm{T}}$ resolution.

The article's information on the experimental momentum resolution for fits with the beam point included (on which all reported Ta results are based) is very scarce. It consists of a mere three points in their Fig. 9. One reads off the resolution $\sigma\left(1 / p_{\mathrm{T}}\right) \sim 0.5(\mathrm{GeV} / c)^{-1}$. This resolution represents the convolution with the non-negligible $\mathrm{d} E / \mathrm{d} x$ resolution, but is hardly compatible with the claimed ${ }^{6}$ $0.3(\mathrm{GeV} / c)^{-1}$.

Incidentally, convincing confirmation that the momentum resolution is much worse than claimed is given in [4] and [5]. Therein, the RPC time-of-flight resolution of $p \sim 200 \mathrm{MeV} / c$ pions that is equivalent to the momentum resolution in the TPC is quoted as $260 \mathrm{ps.} \mathrm{As} \mathrm{succinctly}$ proven in [15], a time-of-flight resolution of $260 \mathrm{ps}$ of pi-

\footnotetext{
5 We conjecture that $1.5 \mathrm{~mm}$ is a realistic guess of the article's average $r \cdot \phi$ precision of TPC cluster coordinates.

6 We note that the dynamic distortions that affect the underlying data set are not specified, and recall that the error of the beam point is not stated. Further, it is surprising that the authors show the momentum resolution of protons for a slice of $\mathrm{d} E / \mathrm{d} x$ but do not show the momentum resolution of protons for a slice of $\beta$ where the contribution from the $\beta$ resolution is much smaller.
} 
Table 1. $\sigma\left(1 / p_{\mathrm{T}}\right)$ with and without the beam point, for three assumptions on the position error of the beam point used in the fit

\begin{tabular}{lcc}
\hline $\begin{array}{l}\sigma \text { of beam } \\
\text { point }[\mathrm{mm}]\end{array}$ & $\begin{array}{c}\sigma\left(1 / p_{\mathrm{T}}\right)\left[(\mathrm{GeV} / c)^{-1}\right] \\
\text { without beam point }\end{array}$ & $\begin{array}{c}\sigma\left(1 / p_{\mathrm{T}}\right)\left[(\mathrm{GeV} / c)^{-1}\right] \\
\text { with beam point }\end{array}$ \\
\hline 1 & 0.54 & 0.27 \\
2 & 0.54 & 0.36 \\
5 & 0.54 & 0.48 \\
\hline
\end{tabular}

ons with $p_{\mathrm{T}}=200 \mathrm{MeV} / c$ is equivalent to a resolution $\Delta p_{\mathrm{T}} / p_{\mathrm{T}}$ of $46 \%$, which is inconsistent by a factor of 4.6 with the claimed $\approx 10 \%$. This gross discrepancy is not mitigated by $10 \%-20 \%$ effects (such as a $\sim 15 \%$ difference between the averages of $p$ and $p_{\mathrm{T}}$ of the used pion sample) that are not taken into account for the sake of clarity.

We note that a good $p_{\mathrm{T}}$ resolution, and its correct understanding, is important for spectral shapes and for charge separation at large $p_{\mathrm{T}}$.

\section{On the particle identification}

The article's pion sample has a large electron background which stems from the conversion of photons from $\pi^{0}$ decay. Electrons can be eliminated by a cut on $\mathrm{d} E / \mathrm{d} x$ up to a momentum of $125 \mathrm{MeV} / c$. In the momentum range $125-250 \mathrm{MeV} / c$ the $\mathrm{d} E / \mathrm{d} x$ of pions and electrons become so close that a separation is not possible. For this very purpose, a system of RPCs had been built with a view to separating pions from electrons by time of flight. As shown in [16], these RPCs have a very good physics performance and permit the separation of pions and electrons in the momentum range $125-250 \mathrm{MeV} / c$.

Rather than using the RPCs for separating pions from electrons in the momentum range $125-250 \mathrm{MeV} / c$, the authors correct for the electron background with the prediction of a Monte Carlo generator while one stated goal of HARP is to check Monte Carlo generators.

Our conjecture that the authors never succeeded calibrating correctly the HARP RPCs stems from their claim of $\mathrm{a} \sim 500 \mathrm{ps}$ timing difference between protons and pions. Such a timing difference is in stark conflict both with established knowledge of RPC signal formation and with our findings from the same RPCs [16]. Although defended by the authors as a new, hitherto unknown, detector physics phenomenon [5,7], it is de facto an artefact stemming from a wrong momentum scale and a wrong momentum resolution ${ }^{7}$. It has been shown in [17] that with a correct momentum scale and a correct momentum resolution no new detector physics phenomenon needs to be invoked.

7 While pions are nearly relativistic and hence their time of flight is insensitive to wrong momentum scale and resolution, protons are not relativistic and hence their time of flight is sensitive to wrong momentum scale and resolution.

\section{The effect on cross-sections}

The three major issues that we hold against the work of the authors are:

- the $p_{\mathrm{T}}$ scale is systematically biased by $\Delta\left(1 / p_{\mathrm{T}}\right) \sim$ $0.3(\mathrm{GeV} / c)^{-1}$;

- the momentum resolution is by a factor of about two worse than claimed;

- the discovery of the ' 500 ps effect' in the HARP RPCs is false.

We consider that a physics analysis based on flawed detector calibrations should not be trusted. Even if, by chance, two or more errors tend to cancel each other, the 'correctness' of results would only stem from the comparison with results from a correct analysis. Possible cancellation of errors is not a theoretical argument: e.g., there are effects that cancel in one magnet polarity but add up in the opposite magnet polarity.

Notwithstanding this, we show in Fig. 1 our measurement of the $q / p_{\mathrm{T}}$ spectrum of tracks from the interactions of $+3 \mathrm{GeV} / c$ protons in a $5 \% \lambda_{\text {abs }}$ Ta target. Each track (charged pions, protons, and background electrons) is entered with its measured $q / p_{\mathrm{T}}$. The virtue of this variable is a constant resolution across the abscissa and that a bias $\Delta\left(1 / p_{\mathrm{T}}\right)$ manifests itself as a horizontal translation. We also show the deformation of this spectrum with a systematic bias of $\Delta\left(1 / p_{\mathrm{T}}\right)=+0.3(\mathrm{GeV} / c)^{-1}$ and with an additional random smearing of $\sigma\left(1 / p_{\mathrm{T}}\right)=0.47(\mathrm{GeV} / c)^{-1}$ on top of the intrinsic $p_{\mathrm{T}}$ resolution of our track reconstruction in the TPC, $\sigma\left(1 / p_{\mathrm{T}}\right)=0.2(\mathrm{GeV} / c)^{-1}[17]$.

The resulting errors of cross-sections depend strongly on the variation of the spectrum with $p_{\mathrm{T}}$. In regions of strong variation, in particular at large $p_{\mathrm{T}}$, cross-sections change by $100 \%$. For $p_{\mathrm{T}}$ below $500 \mathrm{MeV} / c$ (the region cho-

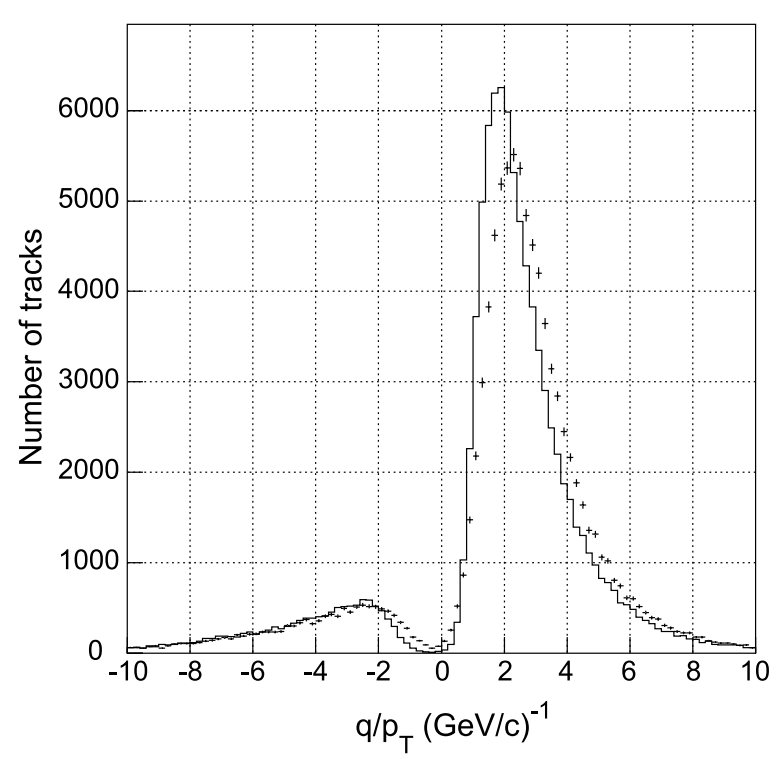

Fig. 1. Measured spectrum of $q / p_{\mathrm{T}}$ from $+3 \mathrm{GeV} / c$ protons interacting in a $5 \% \lambda_{\text {abs }}$ Ta target; the histogram shows our original measurement, the points show the distorted spectrum 
sen by the authors) cross sections still change by $25 \%$ for positive particles, and $15 \%$ for negative particles, in opposite direction. These errors exceed significantly the errors quoted by the authors.

\section{Summary and conclusion}

The two main arguments of the authors that seemingly are in favour of the validity of their work, 'approximate constancy of the average $p_{\mathrm{T}}$ with time in the spill' and 'evidence from the benchmark of protonproton elastic scattering', stem from a wrong analysis concept and are not applicable, respectively. Rather, the authors' $\mathrm{d} E / \mathrm{d} x$ distributions of protons and pions, and their $500 \mathrm{ps}$ timing difference between protons and pions, point to serious defects in the momentum scale and resolution.

The wrong momentum scale and the wrong momentum resolution are not the only reasons, but the dominant ones, why the reported cross-sections should not be trusted.

\section{References}

1. M.G. Catanesi et al., Eur. Phys. J. C 51, 787 (2007)

2. M.G. Catanesi et al., Nucl. Instrum. Methods Phys. Res. A 571, 527 (2007)

3. HARP Collaboration, Nucl. Instrum. Methods Phys. Res. A 571, 564 (2007)

4. M. Bogomilov et al., IEEE Trans. Nucl. Sci. 54, 342 (2007)
5. M. Bogomilov et al., IEEE Trans. Nucl. Sci. 54, 1455 (2007)

6. HARP Collaboration, arXiv:0709:2806 [physics.ins-det] (2007)

7. A. Artamonov et al., arXiv:0709:3756 [physics.ins-det] (2007)

8. F. Dydak, On distortions of TPC coordinates: inhomogeneities of electric and magnetic field, HARP memo 03001, http://cern.ch/dydak/TPCdistortions.ps

9. F. Dydak, A. Krasnoperov, Y. Nefedov, TPC track distortions: correction maps for magnetic and static electric inhomogeneities, HARP Memo 03-002, http://cern.ch/ dydak/TPCdistortions2.ps

10. I. Boyko et al., TPC track distortions III: fiat lux, HARP Memo 05-101, http://cern.ch/dydak/TPCdistortions3.pdf

11. V. Ammosov et al., TPC track distortions IV: post tenebras lux, HARP Memo 07-102, http://cern.ch/dydak/ TPCdistortions4.pdf

12. V. Ammosov et al., The HARP WhiteBook, HARP Memo 06-101, http://cern.ch/dydak/WhiteBook.pdf

13. V. Ammosov et al., Addendum to the HARP WhiteBook, HARP Memo 06-105, http://cern.ch/dydak/ WhiteBookAddendum.pdf

14. V. Ammosov et al., Second Addendum to the HARP WhiteBook, HARP Memo 07-101, http://cern.ch/dydak/ WhiteBookAddendum2.pdf

15. F. Dydak et al., IEEE Trans. Nucl. Sci. 54, 1454 (2007)

16. V. Ammosov et al., Preprint CERN-PH-EP-2007-005, Nucl. Instrum. Methods Phys. Res. A 578, 119 (2007)

17. A. Bolshakova et al., Preprint CERN-PH-EP-2007-030, Nucl. Instrum. Methods Phys. Res. A (accepted), http://cern.ch/dydak/CERN-PH-EP-2007-030.pdf 